\title{
Research on Content Production and Audience Characteristics of Virtual Fan Community
}

-- Taking the Pocket 48 as an Example

\author{
Jinyi $\mathrm{Xu}$ * \\ School of Communication, Fujian Normal University, Fuzhou, China \\ *1173930513@qq.com
}

\begin{abstract}
Fan culture has become a hot topic year by year. In the process of the growth and development of fan culture, it is organically integrated with new media. This article takes SNH48's idol group derivative application Pocket 48 as an example, and uses a case study method to analyze its content production characteristics of instant interaction and two-way emotional resonance. Through the questionnaire method to investigate the characteristics of the audience of the application, the study found that the SNH48 fun-linked virtual community has a decentralized effect on the fan class that has been formed, and enhances user stickiness from the perspective of fan economy.
\end{abstract}

Keywords: Virtual Fan Community; SNH48; Fan Culture; Pocket 48.

\section{Introduction}

\subsection{Background}

The fan community is a synonym for groups with the same interests and values in the same community. With the development of the Internet and new media technologies, the main communication position of the fan community has shifted to social platforms such as Weibo and Douban to form a virtual fan community. SNH48 GROUP is a large-scale female idol group in China that introduced the Japanese AKB model by Shanghai Siba Culture Media Co., Ltd. The main bases of the group are SNH48 (Shanghai), BEJ48 (Beijing), GNZ48 (Guangzhou), and CKG48 (Chengdu). Form an idol cultivation network in China. SNH48 GROUP is different from traditional idols. It focuses on "face-to-face idols" to interact with fans to attract a large number of fan groups. In 2020, the 7th annual idol finals will have a total of 53.35 million voted links (excluding individual fan votes). SNH48 has formed a zero-distance interaction model for fan idols through fixed-frequency theater performances, fan service days, large-scale handshake meetings, and online apps. Pocket 48 app is a unique aggregated virtual fan community for SNH48 fans. According to the survey, Pocket downloads in SNH48 fan groups reached 98.6\%. They are keen on Japanese idols and other funloving groups to carve in Pocket 48. The current research on SNH48 focuses more on the analysis of the "face-to-face" characteristics of its development system, and the localized operation model introduced from the Japanese AKB model. At present, its $\mathrm{O} 2 \mathrm{O}$ online zero-distance communication Pocket 48 is a research blank. defect. Based on this, this article takes Pocket 48 app as an example for analysis.

\subsection{Method}

Taking Pocket 48 of SNH48 as the research object, the content production characteristics of this community are summarized through case study analysis method supplemented by word frequency analysis of Pocket $48 \mathrm{app}$. Use the form of questionnaire to investigate relevant data in the Douban group with strong communication fluidity among fans. Join the research method of participatory observation to analyze the audience characteristics of the SNH48 virtual fan community. 


\section{Results and Discussion}

\subsection{Main Features of Content Production in Virtual Fan Community}

\subsubsection{The Content Presents an Instant Sense of Interaction}

The idols in Pocket 48 interact with fans through the Pocket room. The interface of the Pocket room is similar to the chat window of social software, creating a sense of dialogue in a virtual space. The idol reports what he sees and thinks in real-time in the Pocket room. The main content includes work schedule, live broadcast preview, daily greetings, emotional expression, etc. The SNH48 system pays attention to developing a sense of companionship in the department. Idols only have one performance per week to maintain a fixed exposure. The most convenient and cheapest way for members who do not have a fixed position in the performance is to establish a relationship with fans through a Pocket room. contact. In the survey sample, $72.7 \%$ of users download Pocket 48 because they experience instant interaction with idols. In terms of instant close sensory interaction, $1 \%$ of the samples think that Pocket 48 app has no interactive experience, $10.7 \%$ of the samples have a low score (1-4 points), 74.9\% give a moderately high score (5-8 points), $14.3 \%$ Gave a score of more than 8 points. As a whole, the SNH48 fan community gave a moderate evaluation of the application's online close interaction, with an average score of 6.72 points. The operation mode of the content production of Pocket Room is simple to operate, and it is clear that the audience is around fans who want to get more daily information about the idol stage. According to the member edition data of the 2020 Pocket Report, the idol's commonly used words in the Pocket Room rank first 'everyone', second 'today', and third 'good night'. "Everyone" points to a sense of dialogue. The essence of the application is idol and In the dialogue window of fans, it is easier to get closer by using personal pronouns. Today is generally used for the idol's itinerary report. Will there be participation in today's performance? Today's daily schedule planning, the little idols will tell their fans through the Pocket room. Greetings like good night are generally used in more intimate relationships. The environment of the Pocket room is constantly building a two-way intimate communication space between idols and fans. Idols can pick up their mobile phones between work and use fragmented time to do business. Simple messaging eliminates tedious pre-preparation and post-beautification, and online information exchange breaks the boundaries of time and space, so the presentation of information is instant, The fragmented state creates the feeling that the idol is active around him like a friend.

\subsubsection{Both Sides Develop Emotional Resonance}

The flop function in Pocket 48 is highly sought after by fans, with a usage rate of $68.2 \%$ as a paid function. In the member's Pocket room, fans can send messages to idols at will. Normal messages can be seen by idols in the room but generally will not reply. As long as the flop message is reasonable, idols will provide corresponding replies to the content, and idols will also get corresponding drumsticks. . Take the flop of GNZ48 Zhang Qiong as an example. The original text is as follows.

Fan: I've always said I'm smart since I was a kid, but now I work at a normal job of 965 and earn a good income near home. My friends say that I believe I will be good in the future, but I am not confident. My parents said I was happy, but they didn't live up to their expectations of me. Today's days are relaxed and happy at two o'clock and one line, but I'm not sure whether I've changed and started to enjoy ordinary comfort, or because I'm lazy and mediocre, so I feel comfortable.

Zhang Qiongyu replied: Smart or not is innate and cannot be chosen. The rest of life will depend on one's own choice and experience step by step. As long as you follow your heart and be happy, the expectations of others are for your own good, but you only know if you are really good, and you don't need to be shaken by others. Being smart doesn't necessarily mean living a life full of awards. It only determines whether you can get the awards easily or not. The badge of happiness is granted to yourself. I hope my ideas are useful to you.

There are many flops of this kind, telling about the problems and moods of life finally, raising questions about idol stage performances, comforting idols, etc., to achieve two-way communication and interaction. Traditional idols are so far away. Fans can only learn about idols through third-party 
platforms to experience their works, and there are often great discrepancies between the image created by the media and the person themselves. Comparing the one-way communication between traditional idols and fans and SNH48's original offline handshake meeting, Pocket 48 can accurately convey the message to feel the idol's personal charm. Not only fans get help from idols, idols also receive power from fans. The two sides are communicators and accompany each other to grow and form a strong bond, and generate emotional resonance to cultivate each other. When SNH48 was first introduced from Japan's AKB model, only offline handshake meetings were available. Although face-to-face interaction is possible, offline large-scale handshake meetings have a fixed time and place each year. It is also relatively expensive to purchase a single album to obtain handshake coupons (market price 1210 yuan per minute). Pocket 48's flop function efficiently completes the wording, avoiding the wasted handshake time due to tension offline, and also achieves the effect of emotional resonance.

\subsection{The Characteristics of the Targeted Audience in the Virtual Fan Community}

\subsubsection{Monopoly Information Forms a Gap}

The emergence of Pocket 48 slowly narrowed the distance between idols and fans across the country and even the world through an online path. According to the driving principle of increasing profits in the virtual society, the member idols are regarded as IP commodities, and the idol materials used by the government and the content released by the idols in their Pocket rooms are the main attractions. [1]

Suppose a fan just likes an idol, and only learns about the idol through the idol's Weibo support meeting. In the fan community, it is found that other fans have more information about the idol. There is an information gap effect. Pocket 48 monopolizes the idol's first-hand information. The idol can only broadcast live in the app and talk to fans, otherwise it is a violation of the regulations. Because of Pocket 48's monopoly on members' materials and the inequity of individual information between the Funyuan community, many fans can only follow the idol's dynamics through the materials issued by the support club. Fans must download in order to learn more about idols and communicate with idols.

\subsubsection{Decentralized Fan Groups in Virtual Communities}

Fan groups effectively carry out large-scale collective actions through a 'self-organizing' structure and a more complex mobilization mechanism, and become influential social forces. [2] The fan community usually has opinion leaders who lead the trend of public opinion, spontaneously formed and self-managed support clubs, and manage different things in different levels. They spend a lot of time and energy to support idols and become core fans step by step. Going deep into the phenomenon of collective behavioral income and class mobility of fans to show the behavioral logic of the entire SNH48 Group fan group, its operation is equivalent to a flat real society.[3]For example, SNH48 Xu Yang Yuzhuo's fan support club needs to pay more than the prescribed amount in several investment activities to be eligible to enter the core fan base. The main members support online and offline support, and data stations are anti-black Station work.

The birth of Pocket 48 has played a decentralized role in the original virtual fan community. $71.6 \%$ of the samples will not communicate with other fans when using Pocket 48 , and $57.5 \%$ of users believe that Pocket 48 has the biggest advantage over Weibo Chaohua and other platforms in the Douban community as the solo star chasing resort. Fans have a strong sense of group identity and self-received fans on public platforms. Their speeches are often regarded as the speeches of an entire fan group. Therefore, there are often scolding wars based on fan groups in the Weibo community. Solo chasing stars refers to chasing stars by themselves, not joining the support group, not paying attention to fans, and not seeking concerted action between groups. Pocket 48 dilutes the communication and connection between fans and fans, eliminates the inherent class of fan groups, and fans do not exist as groups. Use your own personal ID to interact with idols, without core fans leading the rhythm of discourse, and without a large number of homogeneous pursuits and comments. Pocket 48 through the interface design greatly reduces the windows that can browse other fan 
information, breaks the fan pyramid structure from the perception, and allows users to experience the more independent star-following mode of developing idols.

\subsubsection{Analyze Uesr Stickiness from the Perspective of Fan Economy}

SNH48's operating model of idols as IP is inseparable from the fan economy. Audiences continue to pay for IP while forming emotional fetters, thereby continuously enhancing their user stickiness. From the perspective of the operating company, Pocket 48 is a corporate media application. The original intention of establishing this application is to promote consumption. Drumsticks are virtual currency in Pocket 48. Users can use Drumsticks to send flop messages to idols, send gifts in live broadcasts, purchase members' surroundings, and participate in voting channels.

According to the questionnaire survey, $69.4 \%$ of users use the payment function in Pocket 48 . Among them, the highest proportion is $87.3 \%$ of users who give gifts through live broadcast. The consumer psychology of fans when giving gifts is to hope that idols will read their ID and say thankful words. The deep-seated psychology is that they are not satisfied with thanks, but hope to be idols. Remember, know that you have been supporting his idol career. In order to be remembered to enhance their sense of existence in front of idols, the idols are familiar with their IDs through gifts. Once you start to consume, you will fall into a psychological cycle, constantly wanting to narrow the distance with idols by increasing economic investment.

Pocket 48 continuously optimizes its service content, enhances the experience and also enhances user stickiness. The topic of the first cup of milk tea in the fall of 2020, which will be hot in the beginning of autumn, has also caused heated discussions in the idol's Pocket room. On the second day, Pocket 48 launched a series of gift special effects for the first cup of milk tea in autumn. Not only keeps pace with the times, but also innovates interesting screen opening voices, and members will be reminded by voice when the application is opened.

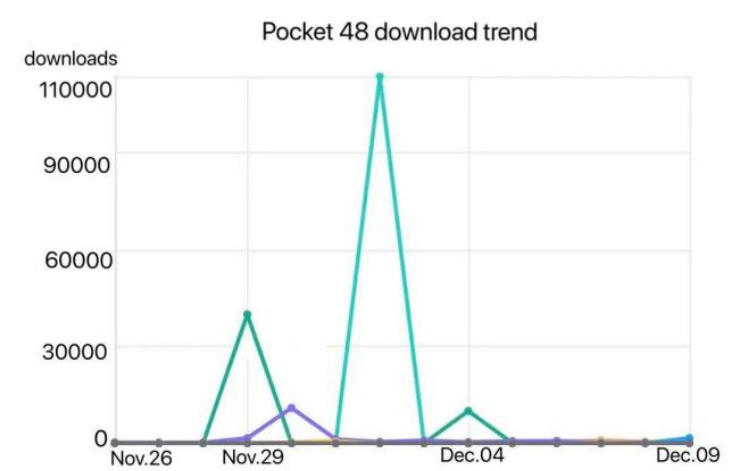

Figure 1. Downloads of Pocket 48 for Android in a single day from November 26 to December 9, 2020

From the data, there are two download peaks on November 29 and December 2, which are the first time the screen opening voice appears, and the second time to update the screen opening voice time. It can be concluded that the continuous optimization of the Pocket 48 system is loved by users. Pocket 48 has added a large number of idol-based products. Although they are non-physical products, they are enough to attract fans to enter the 48-series exclusive application software and consume, and to do their best to increase user stickiness.

\section{Conclusion}

In the information dissemination environment where fan culture is popular, virtual fan communities are gradually occupying an important position in the market. Based on commercial operations combined with new media channels, a two-way communication model for idol fans is formed as an independent discussion space, breaking the original solid fan class structure and creating fun. Equal communication status between groups is easier to generate emotional resonance and 
enhance user stickiness. While the fan virtual community introduces the old and the new, it is necessary to guide and check the content production, and continuously optimize the content production to create a healthy and positive communication environment.

\section{References}

[1] Lan Peng. Introduction to Internet Communication[M]. Beijing: China Renmin University Press, 2001.

[2] Lijin Zhao. Research on the Organizational Structure and Mobilization Mechanism of Fan Community[J], Modern Communication, 2020 (8), 153-157.

[3] Zhihao Ma. Zhongxuan Lin. The collective action logic of the fan community and its class formation -Take the SNH48 Group Fan Support Club as an example[J], Chinese Youth Study, 2018(6), 13-19. 